\title{
A new approach to robust fundamental matrix estimation using an analytic objective function and adjusted gradient projection
}

Cuibing Du, Zongqing Lu, Jing-Hao Xue, Qingmin Liao

Cuibing Du, Zongqing Lu, Jing-Hao Xue, Qingmin Liao, "A new approach to robust fundamental matrix estimation using an analytic objective function and adjusted gradient projection," Proc. SPIE 11179, Eleventh International Conference on Digital Image Processing (ICDIP 2019), 111793D (14 August 2019); doi: $10.1117 / 12.2539648$

Event: Eleventh International Conference on Digital Image Processing (ICDIP 2019), 2019, Guangzhou, China 


\title{
A New Approach to Robust Fundamental Matrix Estimation Using An Analytic Objective Function and Adjusted Gradient Projection
}

\author{
Cuibing $\mathrm{Du}^{\mathrm{a}}$, Zongqing Lu*a, Jing-Hao Xue ${ }^{\mathrm{b}}$, Qingmin Liao ${ }^{\mathrm{a}}$ \\ ${ }^{a}$ Shenzhen Key Lab. Of Information Sci\&Tech, Shenzhen Engineering Lab. Of IS\&DCP \\ Department of Electronics Engineering, Graduate School at Shenzhen, Tsinghua University, China; \\ bUniversity College London, London, UK
}

\begin{abstract}
In this paper we propose a new approach to tackling the challenging problem of robust fundamental matrix estimation from corrupted correspondences. Compared with traditional robust methods, the proposed approach achieves enhanced estimation accuracy and stability. These achievements are attributed mainly to two novelties contributed by the new approach. Firstly, a new, more easily-solvable analytic objective function is proposed to well consider both the presence of correspondence outliers and the computational convenience. Secondly, an adjusted gradient projection method is developed to provide a more stable solver for robust estimation. Experimental results show that the proposed approach performs better than traditional robust methods RANSAC, MSAC, LMEDS and MLESAC, in particular when correspondences were seriously corrupted.
\end{abstract}

Keywords: fundamental matrix, analytic objective function, gradient projection, robust methods.

\section{INTRODUCTION}

In a stereo camera system, the fundamental matrix reflects both of cameras' intrinsic and external parameters ${ }^{[1][2]}$. As an algebraic representation of the epipolar geometry of the system, it plays a crucial role in a large number of computer vision applications, such as 3D construction, feature matching, and structure from motion. Therefore, estimating and optimizing the fundamental matrix, in particular from corrupted correspondences, has attracted considerable research attention in the past few years.

The algorithms to estimate the fundamental matrix can be roughly divided into four types ${ }^{[3][4]}$ : linear methods, distancebased or gradient-based iterative methods, global optimization methods, and robust methods.

The first type of algorithms is linear methods, such as the well-known seven-point ${ }^{[5]}$ and eight-point ${ }^{[6]}$ algorithms and their variants ${ }^{[7][8][9][10]}$. The advantage of this type of methods is that the fundamental matrix can be directly calculated, thus with low computational complexity. However, their accuracies are quite poor when correspondences are corrupted by outliers. The second type of algorithms is iterative methods. The core idea of this group of algorithms is to give each correspondence a weight. The weight can depend on the distances between points and epiploar lines ${ }^{[5][11][12]}$, or be decided by gradients ${ }^{[1][13]}$. Although iterative methods are more accurate than linear methods, they still cannot cope well with outliers. The third type of algorithms is global optimization methods [4][14][15][16]. Although these often computationally-intensive methods perform well under small noise, they also cannot cope well with outliers ${ }^{[2]}$. The fourth type of algorithms is robust methods, which mitigate the effect of outliers through weighting the residual of each correspondence by robust estimators such as the M-estimator [1][17][18]. The RANSAC [5], MSAC [9] and LMEDS [9] algorithms are similar robust methods, based on randomly selecting a set of points to calculate the fundamental matrix by using a linear method, and then choosing the best in terms of certain measures. The MLESAC ${ }^{[19]}$ and MAPSAC ${ }^{[20]}$ both are improved RANSAC. However, these sampling-based methods suffer from several weakness ${ }^{[2]}$, and they cannot guarantee to converge to an optimum in practice especially when the number of outliers is unknown and/or the number of samples is limited. To tackle this, ${ }^{[2]}$ proposed a convex optimization approach to robust estimation of fundamental matrix. It performs well, but the algorithm requires to solve a sequence of semi-definite programming problems, more time-consuming than traditional robust methods.

In this context, by exploiting the advantages of both traditional robust methods and optimization, this paper proposes a new, more accurate, stable and easily-solvable approach, for robust fundamental matrix estimation from corrupted correspondences with outliers. To achieve this, two innovative contributions are made in our proposed approach. Firstly,

Eleventh International Conference on Digital Image Processing (ICDIP 2019) edited by

Jenq-Neng Hwang, Xudong Jiang, Proc. of SPIE Vol. 11179, 111793D @ 2019

SPIE · CCC code: 0277-786X/19/\$21 - doi: 10.1117/12.2539648 
a new, more easily-solvable analytic objective function is proposed to consider well both the presence of correspondence outliers and the computational convenience, such that unlike the sampling-based robust methods, the proposed method can converge to an optimum readily and robustly. Secondly, an adjusted gradient projection method is developed as a more stable solver to solve the constrained optimization problem of robust fundamental matrix estimation.

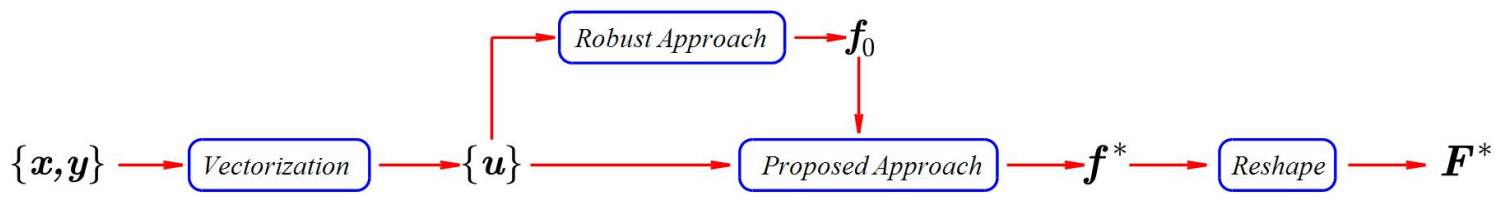

Figure 1. Framework of robust fundamental matrix estimation by the proposal approach.

The framework of robust fundamental matrix estimation by the proposed approach is depicted in Figure.1. It includes four steps: 1) a vectorization step to reform the homogeneous coordinates $\boldsymbol{x}$ and $\boldsymbol{y}$ to their vector version $\boldsymbol{u}$ (see (7a)); 2) a traditional robust-approach step to obtain the initial fundamental matrix $\boldsymbol{F}_{\boldsymbol{0}}$ (or its vector version $\boldsymbol{f}_{\boldsymbol{0}}$ ); 3 ) the proposedapproach step to solve the optimization problem (9) to obtain an optimal solution $\boldsymbol{f}^{*}$ through an improved gradient projection method ${ }^{[21}$; and 4) a reshape step to reform the fundamental matrix from its vector version $f *$ to matrix $F *$.

The rest of the paper is organized as follows. In section 2 , a new analytic objective function is proposed. In section 3 , an adjusted gradient projection method is developed to solve the constrained optimization problem proposed in section 2 . Comparative experiments and results analysis are presented in section 4, and conclusions are drawn in section 5.

\section{A NEW ANALYTIC OBJECTIVE FUNCTION}

\subsection{Problem statement}

Given a pair of images of the same scene from two cameras, the fundamental matrix $\boldsymbol{F}$ satisfies the epipolar constraint

$$
\boldsymbol{y}^{T} F \boldsymbol{x}=0
$$

where $\boldsymbol{x}=\left(u_{x}, v_{x}, 1\right)^{T}$ and $\boldsymbol{y}=\left(u_{y}, v_{y}, 1\right)^{T}$ are the homogeneous coordinates of the corresponding points that the same $3 \mathrm{D}$ point projected in two images. The fundamental matrix $\boldsymbol{F}$ itself can be decomposed as

$$
F=K^{-T}(t \times R) K^{-1}
$$

where $\boldsymbol{K}$ is the camera intrinsic matrix; vector $\boldsymbol{t}$ and matrix $\boldsymbol{R}$ express the translation and rotation of the cameras, respectively; the operator $\times$ express the cross operation. Hence if $\boldsymbol{t} \neq 0$, matrix $\boldsymbol{F}$ is of rank deficit, i.e. the determinant of $\boldsymbol{F}$ is zero. In addition, to eliminate the influence of the modulus of $\boldsymbol{F}$ and avoid trivial solution $\boldsymbol{F}=\boldsymbol{0}, \boldsymbol{F}$ is normalized. Then problem (1) can be rewritten as

$$
\begin{aligned}
& \boldsymbol{y}^{\boldsymbol{T}} \boldsymbol{F} \boldsymbol{x}=\mathbf{0} \\
& \operatorname{det}(\boldsymbol{F})=0 \\
& \|\boldsymbol{F}\|_{F}^{2}=0
\end{aligned}
$$

\subsection{A new analytic objective function}

\subsubsection{Using analytic mapping $m(\cdot)$}




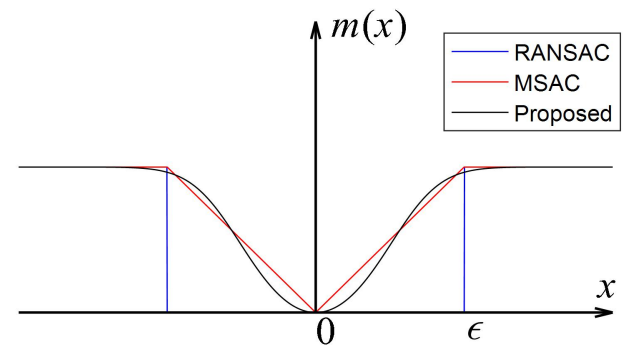

Figure 2. Mapping functions.

Because of the errors from feature extraction and outliers, requirement (3) is not satisfied in most cases. Hence, $\beta$-norm $\left\|\boldsymbol{y}^{\boldsymbol{T}} \boldsymbol{F} \boldsymbol{x}\right\|_{\beta}$ is used instead as the loss from estimating fundamental matrix $\boldsymbol{F}$. Traditionally, $\beta$ is set to 1 . Moreover, to reduce the influence of outliers on the $\beta$-norm, following the strategies in robust statistics, RANSAC ${ }^{[5]}$ and MSAC ${ }^{[9]}$ construct a mapping function $m(\cdot)$ to shrink large losses, as illustrated in Figure.2. As $m(\cdot)$ is an even function, the estimation of $\boldsymbol{F}$ from $\mathrm{N}$ correspondences can be rewritten as the following optimization problem:

$$
\begin{array}{ll}
\min & \sum_{i=1}^{N} m\left(y_{i}^{T} F x_{i}\right) \\
\text { s.t. } & \operatorname{det}(F)=0, \\
& \|F\|_{F}^{2}=1 .
\end{array}
$$

For sampling-based traditional robust methods, such as RANSAC ${ }^{[5]}$, MSAC ${ }^{[9]}$, LMEDS ${ }^{[9]}$ and MLESAC ${ }^{[19]}$, an approximate fundamental matrix is obtained by a linear method like the eight-point algorithm ${ }^{[6]}$ from a set of sample points, and repeating this can attain a set of matrices, from which the matrix with the smallest loss is selected. However, this approximate solution may not reach the optimum of problem (6) (8). Moreover, the mapping functions $m(\cdot)$ of RANSAC and MSAC are not analytic functions, hence gradient-based methods cannot be directly applied.

To solve these problems, we propose an analytic mapping

$$
m(x)=\frac{e^{\sigma x^{2}}-e^{-\sigma x^{2}}}{e^{\sigma x^{2}}+e^{-\sigma x^{2}}} .
$$

Our proposal is an analytic approximate to the $m(\cdot)$ of MSAC ${ }^{[9]}$ (see the black curve in Figure.2), so that problem (6) (8) can be easily solved by gradient-based methods.

\subsubsection{Using angle loss $\theta$}

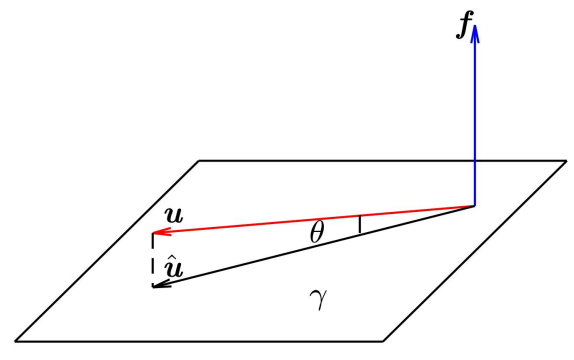

Figure 3. Vector relationship. 
The error function $\left\|\boldsymbol{y}^{\boldsymbol{T}} \boldsymbol{F} \boldsymbol{x}\right\|_{\beta}$ is affected by the position of the correspondence. To mitigate this effect, normalizing the correspondence can be considered. To illustrate better, we rewrite (1) in a linear form as that in linear methods:

$$
u^{T} f=0,
$$

where $\boldsymbol{u}$ is a combinatorial representation of the correspondence between $\boldsymbol{x}$ and $\boldsymbol{y}$, and $\boldsymbol{f}$ is a vector version of fundamental matrix $\boldsymbol{F}$ :

$$
\begin{aligned}
& \boldsymbol{u}=\left(u_{y} u_{x}, v_{y} u_{x}, u_{x}, u_{y} v_{x}, v_{y} v_{x}, v_{x}, u_{y}, v_{y}, 1\right)^{T}, \\
& \boldsymbol{f}=\left(F_{11}, F_{21}, F_{31}, F_{12}, F_{22}, F_{32}, F_{13}, F_{23}, F_{33},\right)^{T} .
\end{aligned}
$$

Equation (10) indicates that vectors $\boldsymbol{u}$ and $\boldsymbol{f}$ should be perpendicular to each other, which is however not so given the loss. As illustrated in Figure.3, $\gamma$ is the super-plane perpendicular to vector $\boldsymbol{f} ; \hat{\boldsymbol{u}}$ is the projection of vector $\boldsymbol{u}$ on super-plane $\gamma$; and $\theta$ is the angle between vectors $\boldsymbol{u}$ and $\hat{\boldsymbol{u}}$. Ideally, $\boldsymbol{u}$ should coincident with $\hat{\boldsymbol{u}}$, that is, the angle $\theta$ should be zero. But in practice due to corrupted correspondence, $\theta$ is not zero for most correspondences. Hence the angle $\theta$ itself can be used as a surrogate of the normalized loss, which enjoys the advantage of not being affected by the modulus of $\boldsymbol{u}$ :

$$
\theta(\boldsymbol{u}, \boldsymbol{f})=\arcsin \left(\frac{\boldsymbol{u}^{\boldsymbol{T}} \boldsymbol{f}}{\|\boldsymbol{u}\| \boldsymbol{f} \|}\right)
$$

\subsubsection{New constrained optimization problem}

After proposing a new mapping function (9) and a new error function (13), now we can build a new objective function for the constrained optimization problem (6) (8):

$$
\begin{array}{ll}
\min _{\boldsymbol{f} \in \boldsymbol{S}} & P(\boldsymbol{f}) \\
\text { s.t. } & Q_{i}(\boldsymbol{f})=0(i=1,2),
\end{array}
$$

where $\boldsymbol{S}$ denotes the intersecting super-surface of constraints $Q_{1}(\boldsymbol{f})=\boldsymbol{0}$ and $Q_{2}(\boldsymbol{f})=\boldsymbol{0}$, and

$$
\begin{gathered}
P(\boldsymbol{f})=\sum_{i=1}^{N} m\left(\theta\left(\boldsymbol{u}_{\boldsymbol{i}}, \boldsymbol{f}\right)\right), \\
Q_{1}(\boldsymbol{f})=f_{1}\left(f_{5} f_{9}-f_{6} f_{8}\right)-f_{2}\left(f_{4} f_{9}-f_{6} f_{7}\right)+f_{3}\left(f_{4} f_{8}-f_{5} f_{7}\right), \\
Q_{2}(\boldsymbol{f})=\sum_{i=1}^{N} f_{i}^{2}-1 .
\end{gathered}
$$

\section{AN ADJUSTED GRADIENT PROJECTION METHOD}

\subsection{A gradient projection (GP) method}

Since both $P(\boldsymbol{f})$ and $Q_{i}(\boldsymbol{f})$ are complex functions, it is not easy to apply the KKT conditions to solve the constrained optimization problem (14) (15). Here we adjust a gradient projection method [21] to fit for the characteristics of fundamental matrix, with its initial solution $\boldsymbol{f}_{\boldsymbol{0}}$ obtained by a traditional robust method like RANSAC. 


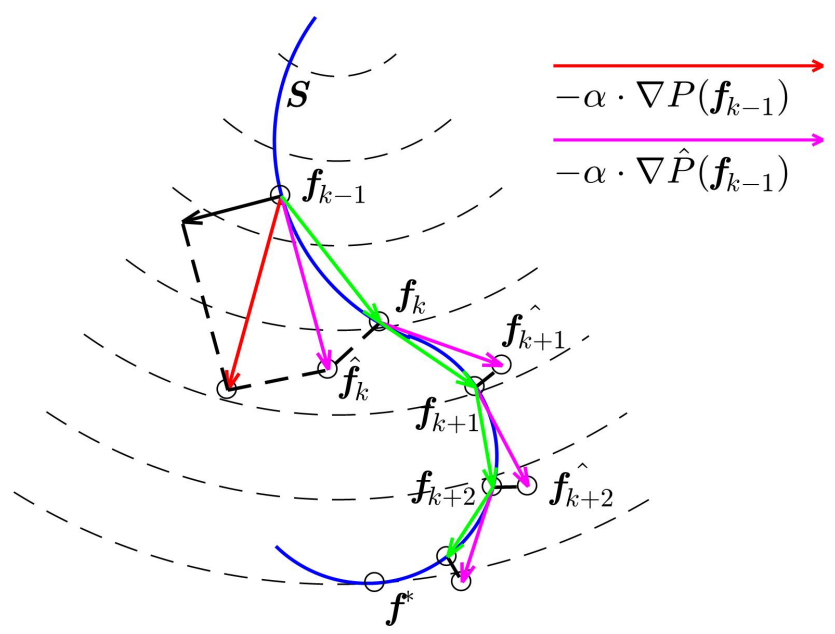

Figure 4. A gradient projection method.

We first introduce the gradient projection method $\left.{ }^{[21}\right]$ and illustrate its iterative process in Figure.4. Each of its iterations (from $\boldsymbol{f}_{k-1}$ to $\boldsymbol{f}_{k}$ ) consists of three steps: 1) projecting gradient $\nabla P\left(\boldsymbol{f}_{k-1}\right)$ (red arrow in Figure.4) of objective function $P\left(\boldsymbol{f}_{k-1}\right)$ to the tangent direction $\nabla \hat{P}\left(\boldsymbol{f}_{k-1}\right)$ (magenta arrow) of the constraints super-surface $\boldsymbol{S} ; 2$ ) gradient descending along the tangent direction $\nabla \hat{P}\left(\boldsymbol{f}_{k-1}\right)$ of $\boldsymbol{S}$ from point $\boldsymbol{f}_{k-1}$ to point $\hat{\boldsymbol{f}}_{k}$; and 3) projecting point $\hat{\boldsymbol{f}}_{k}$ to point $\boldsymbol{f}_{k}$ on $\boldsymbol{S}$ to satisfy the constraints. The mathematical expressions corresponding to these three steps are

$$
\begin{aligned}
& \nabla \hat{P}\left(\boldsymbol{f}_{k-1}\right)=L\left[\nabla P\left(\boldsymbol{f}_{k-1}\right)\right], \\
& \hat{\boldsymbol{f}}_{k}=\boldsymbol{f}_{k-1}-\alpha \cdot \nabla \hat{P}\left(\boldsymbol{f}_{k-1}\right), \\
& \boldsymbol{f}_{k}=C\left(\hat{\boldsymbol{f}}_{\boldsymbol{k}}\right),
\end{aligned}
$$

where $L(\cdot)$ and $C(\cdot)$ are the projection functions required in step 1 and step 3, respectively, which need to be well adjusted to our robust fundamental matrix estimation problem.

\subsection{Adjusting the GP method}

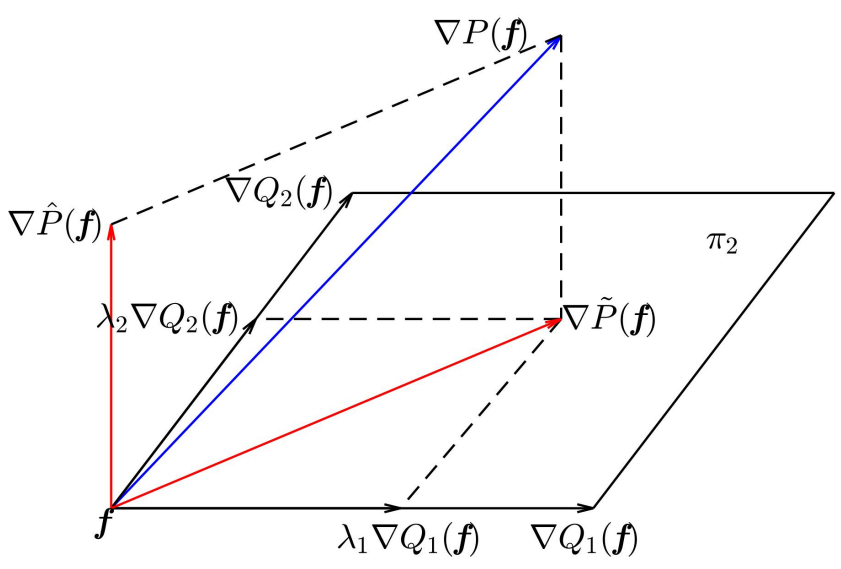

Figure 5. Implementation of projection function $L(\cdot)$. 


\subsubsection{Finding $L(\cdot)$}

The projection function $L(\cdot)$ is to project gradient $\nabla P(\boldsymbol{f})$ of objective function $P(\boldsymbol{f})$ to the tangent super-plane ( $\left.\pi_{1}\right)$ of $\boldsymbol{S}$ at point $\boldsymbol{f}$, and the resultant projection is $\nabla \hat{P}(\boldsymbol{f})$. To simplify calculation, suppose the projection of gradient to the vertical super-plane $\left(\pi_{2}\right)$ at point $\boldsymbol{f}$ is $\nabla \widetilde{P}(\boldsymbol{f})$, where $\pi_{2}$ is defined by the normal direction of supersurface $\boldsymbol{S}$ at $\boldsymbol{f}$. Because

$$
\nabla \hat{P}(\boldsymbol{f})+\nabla \widetilde{P}(\boldsymbol{f})=\nabla P(\boldsymbol{f})
$$

attaining $\nabla \hat{P}(\boldsymbol{f})$ is equivalent to obtaining the projection $\nabla \widetilde{P}(\boldsymbol{f})$ on super-plane $\pi_{2}$. Then as shown in Figure.5, to obtain $\nabla \widetilde{P}(\boldsymbol{f})$, we only need to build an optimization problem as

$$
\min _{\lambda=\left\{\lambda_{1}, \lambda_{2}\right\}}\|\nabla P(\boldsymbol{f})-\nabla \widetilde{P}(\boldsymbol{f})\|_{F}^{2},
$$

where the normal direction $\nabla \widetilde{P}(\boldsymbol{f})=\sum_{i=1}^{2} \lambda_{i} \cdot \nabla Q_{i}(\boldsymbol{f})$, with $Q_{i}(\boldsymbol{f})=0$ the constraints to build $\boldsymbol{S}$. This problem can be simply solved by the method of least squares (LS).

\subsubsection{Approximating $C(\cdot)$}

In step 3 we need to use the projection function $C(\cdot)$ to project $\hat{\boldsymbol{f}}_{k}$ to a point $\boldsymbol{f}_{k}$ on the constraints super-surface $\boldsymbol{S}$. Instead of projecting along the super-plane $\pi_{2}$, we utilize an adjusted approach to projecting $\hat{\boldsymbol{f}}_{k}$ to the closest point on $\boldsymbol{S}$, which is equivalent to solve the following optimization problem:

$$
\min _{f_{k} \in \boldsymbol{S}}\left\|\boldsymbol{f}_{\boldsymbol{k}}-\hat{\boldsymbol{f}}_{\boldsymbol{k}}\right\|_{F}^{2} .
$$

Since $\boldsymbol{f}$ is a 9-dimensional vector, implementing $C(\cdot)$ by solving optimization problem (24) is not easy. Hence here we realize this by adjusting an approximate method ${ }^{[1]}$ to the characteristics of fundamental matrix. More specifically, we first transform $\hat{\boldsymbol{f}}_{k}$ to satisfy the constraint (7) by applying singular value decomposition (SVD) to the fundamental matrix $\boldsymbol{F}$ reshaped from $\hat{\boldsymbol{f}}_{k}$, shrinking the minimum singular value to zero to ensure rank deficient, and restoring $\boldsymbol{F}$. Then we normalize $\boldsymbol{F}$ to satisfy the constraint (8).

By developing this adjusted approximate method involving no gradient descent, and projecting along the shortest path from $\hat{\boldsymbol{f}}_{k}$ to $\boldsymbol{f}_{k}$, we reduce the risk of unstable projection to an opposite direction due to any local optimum on the path. Combining the implementations of $L(\cdot)$ and $C(\cdot)$, the algorithm of our proposed approach is shown in Algorithm 1.

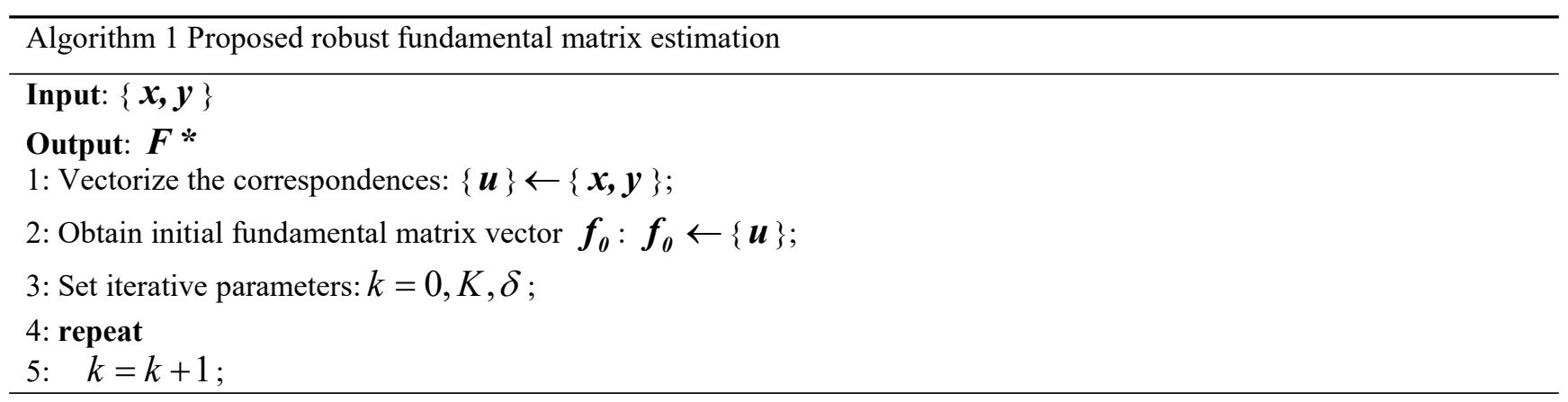


6: Calculate $\nabla P(\boldsymbol{f})$ and $\nabla Q_{i}(\boldsymbol{f})$ at point $\boldsymbol{f}_{\boldsymbol{k}-1}$;

7: $\quad$ Estimate parameters $\lambda$ by (13): $\lambda \leftarrow\left(\nabla P(\boldsymbol{f}), \quad \nabla Q_{i}(\boldsymbol{f})\right)$;

8: Calculate projected gradient $\nabla \hat{P}(\boldsymbol{f})$ at point $\boldsymbol{f}_{\boldsymbol{k}-1}: \nabla \hat{P}(\boldsymbol{f})=\nabla P(\boldsymbol{f})-\sum_{i=1}^{2} \lambda_{i} \cdot \nabla Q_{i}(\boldsymbol{f})$;

9: Update $\hat{\boldsymbol{f}}_{\boldsymbol{k}}: \hat{\boldsymbol{f}}_{\boldsymbol{k}}=\boldsymbol{f}_{\boldsymbol{k}-1}-\alpha \cdot \nabla \hat{P}(\boldsymbol{f})$;

10: Reshape: $\boldsymbol{F} \leftarrow \hat{\boldsymbol{f}}_{\boldsymbol{k}}$;

11: Singular value decomposition for $\boldsymbol{F}:[\boldsymbol{U}, \boldsymbol{D}, \boldsymbol{V}]=S V D(\boldsymbol{F})$;

12: Set the minimum singular value zero: $D_{33}=0$;

13: Inverse singular value decomposition to restore $\boldsymbol{F}: \boldsymbol{F}=\boldsymbol{U D} \boldsymbol{V}^{\boldsymbol{T}}$;

14: Normalization: $\boldsymbol{F}=\boldsymbol{F} /\|\boldsymbol{F}\|_{F}$;

15: Reshape: $\boldsymbol{f}_{\boldsymbol{k}} \leftarrow \boldsymbol{F}$;

16:until $k>K$ or $\left|\boldsymbol{f}_{\boldsymbol{k}}-\boldsymbol{f}_{\boldsymbol{k}-1}\right|<\delta$;

17: Reshape to obtain optimal solution: $F^{*} \leftarrow \boldsymbol{f}^{*}$

\section{EXPERIMENTS}

\subsection{Datasets}

In the experiments, we use both synthetic and real datasets to verify the efficiency of the proposed approach.

The synthetic dataset is generated by the following steps: 1) generating a 3D scene set $\{X\}$ consisting of 400 points distributed randomly; 2) randomly setting the camera translation $(\boldsymbol{t})$, rotation $(\boldsymbol{R})$ and intrinsic parameter $(\boldsymbol{K}) ; 3$ ) generating correspondences $\{(\boldsymbol{x}, \boldsymbol{y})\}$ by projecting $\{X\}$ to both cameras; 4) selecting 200 correspondences from $\{(\boldsymbol{x}, \boldsymbol{y})\}$ as the test set; 5) selecting another 100 correspondences as the training set.

The real dataset that we used is VGG, University of Oxford. It contains six pairs of stereo images: House, Library, Wadham, Merton I, Merton II and Merton III.

\subsection{Experimental protocol}

Experimental set-up: For each pair of images from the VGG or synthetic dataset, we ran seven sets of experiments, with 10-70 training correspondences randomly picked and corrupted by large Gaussian noise (i.e. outlier number $N_{o}=10-70$ ), respectively. Each set of experiments was run 50 times.

Performance measures: We compared different robust methods in terms of two performance measures on the test set:

$$
\begin{aligned}
& \text { Absolute Error }=\sum_{i=1}^{N}\left|\boldsymbol{y}_{\boldsymbol{i}}^{\boldsymbol{T}} \boldsymbol{F} \boldsymbol{x}_{\boldsymbol{i}}\right|, \\
& \text { Angle Error }=\sum_{i=1}^{N}\left|\theta\left(\boldsymbol{u}_{\boldsymbol{i}}, \boldsymbol{f}\right)\right| .
\end{aligned}
$$




\subsection{Experimental results and analysis}

Table 1. Mean(and standard deviationi) of absolute error. Table 2. Mean(and standard deviationi) of angle error.

\begin{tabular}{c|c|c|c|c|c}
\hline$N_{o}$ & RANSAC & MSAC & LMEDS & MLESAC & Proposed \\
\hline \hline \multirow{2}{*}{10} & 1.8067 & 1.7654 & 1.7590 & 1.8319 & $\mathbf{1 . 7 2 5 8}$ \\
& $(0.1408)$ & $(0.0967)$ & $(0.0875)$ & $(0.1533)$ & $(\mathbf{0 . 0 8 2 2})$ \\
\hline \multirow{2}{*}{20} & 1.8678 & 1.7874 & 1.7758 & 1.8620 & $\mathbf{1 . 7 6 0 4}$ \\
& $(0.1488)$ & $(0.0883)$ & $(0.0909)$ & $(0.1979)$ & $(\mathbf{0 . 1 0 3 0})$ \\
\hline \multirow{2}{*}{30} & 1.8736 & 1.8143 & 1.8279 & 1.8979 & $\mathbf{1 . 7 8 0 9}$ \\
& $(0.1357)$ & $(0.1025)$ & $(0.0908)$ & $(0.2092)$ & $(\mathbf{0 . 1 2 2 7})$ \\
\hline \multirow{2}{*}{40} & 1.9381 & 1.8791 & 1.8693 & 1.9435 & $\mathbf{1 . 7 9 8 8}$ \\
& $(0.1740)$ & $(0.1434)$ & $(0.1173)$ & $(0.2435)$ & $(\mathbf{0 . 1 2 5 6})$ \\
\hline \multirow{2}{*}{50} & 1.9854 & 1.9600 & 1.9593 & 1.9817 & $\mathbf{1 . 8 6 8 8}$ \\
& $(0.1608)$ & $(0.1884)$ & $(0.1790)$ & $(0.2780)$ & $(\mathbf{0 . 1 5 6 3})$ \\
\hline \multirow{2}{*}{60} & 2.2091 & 2.1622 & 2.2472 & 2.1689 & $\mathbf{2 . 0 1 7 4}$ \\
& $(0.3603)$ & $(0.3501)$ & $(0.4676)$ & $(0.4404)$ & $(\mathbf{0 . 2 6 6 0})$ \\
\hline \multirow{2}{*}{70} & 2.7397 & 2.7328 & 2.8324 & 2.7276 & $\mathbf{2 . 3 8 2 3}$ \\
& $(0.8556)$ & $(0.1268)$ & $(0.9012)$ & $(0.8022)$ & $(\mathbf{0 . 7 5 7 9})$ \\
\hline
\end{tabular}

\begin{tabular}{c|c|c|c|c|c}
\hline$N_{o}$ & RANSAC & MSAC & LMEDS & MLESAC & Proposed \\
\hline \hline \multirow{2}{*}{10} & 1.1853 & 1.1445 & 1.1309 & 1.1522 & $\mathbf{1 . 1 0 9 3}$ \\
& $(0.1184)$ & $(0.0753)$ & $(0.0615)$ & $(0.1185)$ & $(\mathbf{0 . 0 6 2 6})$ \\
\hline \multirow{2}{*}{20} & 1.2310 & 1.1630 & 1.1458 & 1.1828 & $\mathbf{1 . 1 3 3 5}$ \\
& $(0.1294)$ & $(0.0772)$ & $(0.0713)$ & $(0.1466)$ & $(\mathbf{0 . 0 8 5 3})$ \\
\hline \multirow{2}{*}{30} & 1.2324 & 1.1769 & 1.1938 & 1.2044 & $\mathbf{1 . 1 5 6 9}$ \\
& $(0.1135)$ & $(0.0784)$ & $(0.0740)$ & $(0.1438)$ & $(\mathbf{0 . 0 9 7 1})$ \\
\hline \multirow{2}{*}{40} & 1.2639 & 1.2226 & 1.2140 & 1.2187 & $\mathbf{1 . 1 7 4 1}$ \\
& $(0.1245)$ & $(0.0972)$ & $(0.0808)$ & $(0.1609)$ & $(\mathbf{0 . 0 9 2 8})$ \\
\hline \multirow{2}{*}{50} & 1.2953 & 1.2736 & 1.2746 & 1.3082 & $\mathbf{1 . 2 1 5 5}$ \\
& $(0.1130)$ & $(0.1265)$ & $(0.1187)$ & $(0.1775)$ & $(\mathbf{0 . 1 1 1 6})$ \\
\hline \multirow{2}{*}{60} & 1.4398 & 1.4059 & 1.4673 & 1.4393 & $\mathbf{1 . 3 0 8 9}$ \\
& $(0.2227)$ & $(0.2287)$ & $(0.2953)$ & $(0.2526)$ & $(\mathbf{0 . 1 7 4 2})$ \\
\hline \multirow{2}{*}{70} & 1.7834 & 1.7570 & 1.8336 & 1.7880 & $\mathbf{1 . 5 3 9 8}$ \\
& $(0.5559)$ & $(0.6868)$ & $(0.5669)$ & $(0.5426)$ & $(\mathbf{0 . 4 6 0 8})$ \\
\hline
\end{tabular}

In the experiments we compare the performance of the proposed approach with other robust methods including RANSAC [5], MSAC [9], LMEDS [9] and MLESAC [19]. For all these methods, the number of iterations was set to 500, and in each iteration the fundamental matrix was calculated by using the eight-point method ${ }^{[6]}$.

The results on the synthetic dataset are summarized in Tables 1-2, where the proposed approach performs the best (in bold), with both the smallest mean of errors, indicating that the estimation accuracy of fundamental matrix is improved, and the smallest variance of errors, indicating that the estimation stability is also improved. The improvement is generally more remarkable when the number of outliers increases.

Some results on the real dataset areshown in Figures.6-7, which clearly indicate that the proposed approach performs consistently better than the compared methods. Once again, with the proportion of outliers increasing, the gap between the methods becomes larger, indicating the improvement induced by the proposed method becomes more substantial.
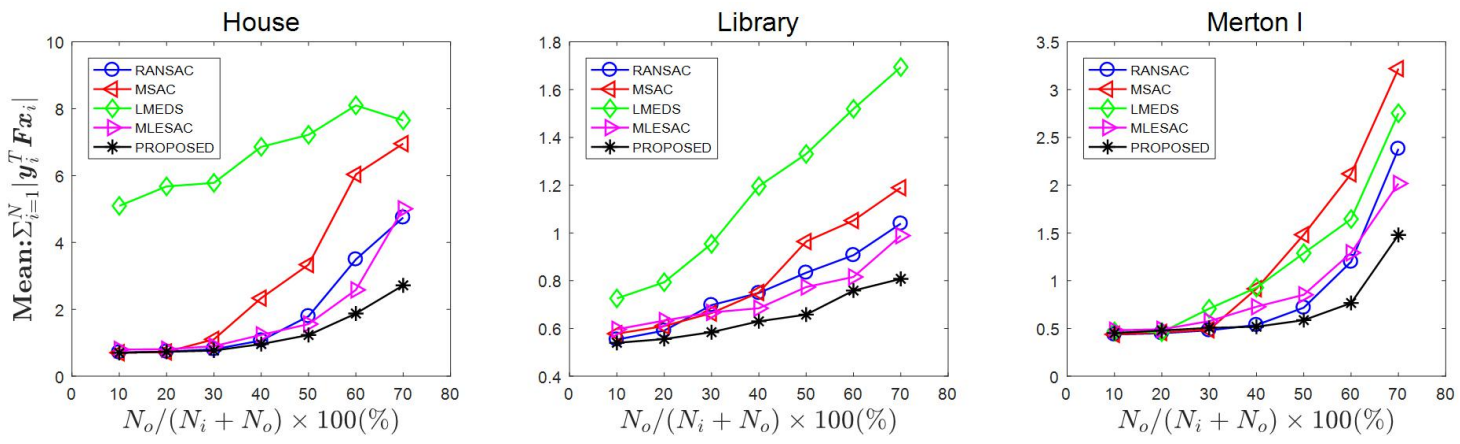

Figure 6. Absolute error on the VGG dataset.
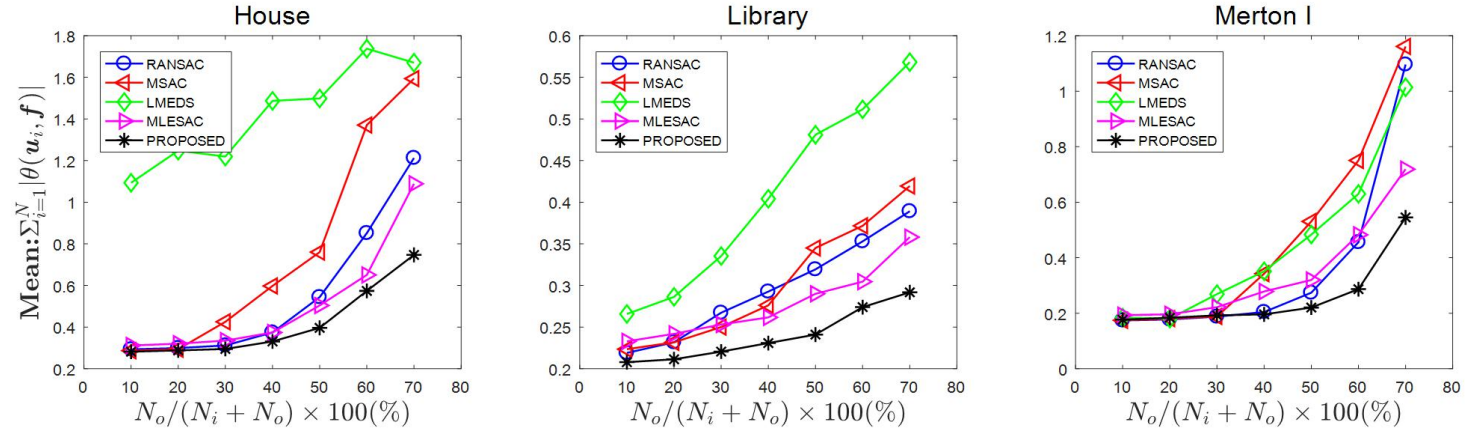

Figure 7. Angle error on the VGG dataset. 


\section{CONCLUSIONS}

This paper proposed a new approach to robust fundamental matrix estimation from corrupted correspondences. In order to improve the accuracy and stability of robust fundamental matrix estimation, a new analytic objective function has been proposed and an adjusted gradient projection method is developed. Experiment results on both synthetic and real datasets demonstrate that the proposed approach performs better than traditional robust methods, particularly when the corruption in correspondences is serious.

\section{REFERENCES}

[1] Richard Hartley and Andrew Zisserman, Multiple View Geometry in Computer Vision, Cambridge University Press, 2003.

[2] Cheng. Y, Lopez. J. A, Camps. O, and Sznaier. M, "A convex optimization approach to robust fundamental matrix estimation," in IEEE Conference on Computer Vision and Pattern Recognition, pp. 2170-2178, 2015.

[3] Xavier Armangu'e and Joaquim Salvi, "Overall view regarding fundamental matrix estimation," Image \& Vision Computing, vol. 21, no. 2, pp. 205-220, 2003.

[4] Florian Bugarin, Adrien Bartoli, Didier Henrion, Jean Bernard Lasserre, Jean Jos Orteu, and Thierry Sentenac, "Rank-constrained fundamental matrix estimation by polynomial global optimization versus the eightpoint algorithm," Journal of Mathematical Imaging \& Vision, vol. 53, no. 1, pp. 1-19, 2015.

[5] Zhengyou Zhang, "Determining the epipolar geometry and its uncertainty: A review," International Journal of Computer Vision, vol. 27, no. 2, pp. 161-195, 1998.

[6] Wojciech Chojnacki, Michael J. Brooks, Anton Van Den Hengel, and Darren Gawley, "Revisiting Hartley's normalized eight-point algorithm," IEEE Transactions on Pattern Analysis \& Machine Intelligence, vol. 25, no. 9, pp. 1172-1177, 2003.

[7] Longuet-Higgins. H. C, "A computer algorithm for reconstructing a scene from two projections," Nature, vol. 293, no. 5828 , pp. $133-135,1981$.

[8] Quan Tuan Luong and Olivier D. Faugeras, "The fundamental matrix: Theory, algorithms, and stability analysis," International Journal of Computer Vision, vol. 17, no. 1, pp. 43-75, 1996.

[9] Torr. H. S and Murray. D. W, "The development and comparison of robust methods for estimating the fundamental matrix," International Journal of Computer Vision, vol. 24, no. 3, pp. 271-300, 1997.

[10] Tsuyoshi Migita and Takeshi Shakunaga, "Evaluation of epipole estimation methods with/without rank-2 constraint across algebraic/geometric error functions," in Computer Vision and Pattern Recognition, 2007. CVPR'07. IEEE Conference on, pp. 1-7, 2007.

[11] Joaquim Salvi Mas, An Approach to coded structured light to obtain three dimensional information, Ph.D. thesis, Universitat de Girona, 2001.

[12] Karthik Mohan and Maryam Fazel, Iterative reweighted algorithms for matrix rank minimization, JMLR.org, 2012.

[13] Wojciech Chojnacki, Michael J. Brooks, Anton Van Den Hengel, and Darren Gawley, "A new approach to constrained parameter estimation applicable to some computer vision problems," Statistical Methods in Video Processing Workshop, 2007.

[14] Yasuyuki Sugaya and Kenichi Kanatani, "High accuracy computation of rank-constrained fundamental matrix by efficient search," in British Machine Vision Conference 2007, pp. 17-24, 2007.

[15] Yinqiang Zheng, Sugimoto.S, and Okutomi.M, "A branch and contract algorithm for globally optimal fundamental matrix estimation," in Computer Vision and Pattern Recognition, pp. 2953-2960, 2011.

[16] Yinqiang Zheng, Shigeki Sugimoto, and Masatoshi Okutomi, "A practical rank-constrained eight-point algorithm for fundamental matrix estimation," in Proceedings of the IEEE Conference on Computer Vision and Pattern Recognition, pp. 1546-1553, 2013.

[17] Peter J. Huber, Robust Statistics, Wiley, 2011.

[18] John Wilder, Data analysis and regression, AddisonWesley Pub. Co., 1977.

[19] Torr. P. H. S and Zisserman. A, "MLESAC: A new robust estimator with application to estimating image geometry," Computer Vision \& Image Understanding, vol.78, no. 1, pp. 138-156, 2000.

[20] Torr. P. H. S, "Bayesian model estimation and selection for epipolar geometry and generic manifold fitting," International Journal of Computer Vision, vol. 50, no. 1, pp. 35-61, 2002.

[21] David G. Luenberger and Yinyu Ye, Linear and Nonlinear Programming, Springer, 2015. 